\title{
Avaliação Qualitativa do ProLearn4ALL: um Projeto de Investigação Baseado na Resolução de Problemas
}

\author{
Catarina Mangas, Carla Freire \& Olga Santos
}

Resumo:

O ProLearn4ALL - Maletas Pedagógicas para TODOS é um projeto que tem como principal propósito a criação de recursos lúdico-pedagógicos acessíveis a crianças do $1^{\circ}$ Ciclo do Ensino Básico, com o intuito de as sensibilizar para a diferença e, em particular, para os quatro domínios da deficiência (intelectual, motora, auditiva e visual). Este tem a particularidade de incluir, na sua equipa de trabalho, diversos elementos, não só docentes e investigadores, mas também estudantes de diferentes níveis do Ensino Superior (Cursos Técnicos Superiores Profissionais, Licenciaturas e Mestrados) que são confrontados com uma sequência de questões para as quais têm de encontrar respostas e soluções válidas. A aprendizagem e investigação baseada em problemas tornaram-se, portanto, as estratégias seguidas ao longo deste trabalho, estratégias estas que implicam uma avaliação sistemática. A avaliação de todo o processo, desde a fase de planificação à implementação, encontra-se descrita neste artigo, refletindo-se sobre as opções tomadas ao nível dos instrumentos e técnicas a aplicar. Estando este projeto em desenvolvimento, espera-se que os resultados desta avaliação permitam compreender as repercussões do projeto nos conhecimentos e atitudes dos estudantes do Ensino Superior, mas também o impacte do mesmo em turmas do Ensino Básico, que incluem crianças com e sem deficiência, e dos seus respetivos professores.

Palavras-chave:

prolearn4all; avaliação qualitativa; inclusão; $1^{\circ}$ Ciclo do Ensino Básico; recursos lúdico-pedagógicos. 


\title{
Qualitative Assessment of the ProLearn4ALL: a Research Project based on Problem Solving
}

\begin{abstract}
The ProLearn4ALL - Maletas Pedagógicas para TODOS is a project with the main purpose of creating educational and ludic resources that are accessible to children in Primary Schools, in order to raise awareness of the difference and, in particular, of the four disability areas (intellectual, motor, auditory and visual). This has the particularity of its work team including several members, not only teachers and researchers, but also students from different levels of Higher Education (Professional Technical Higher Education Courses, Bachelors and Masters degrees) who are confronted with a sequence of questions for which they have to find valid answers and solutions. Problem-based learning has become the strategy pursued throughout the research and student's training, a strategy which implies a systematic evaluation of the work developed. The evaluation of the entire process, from the planning to the implementation phase, is described in this article, reflecting on the options taken at the level of the instruments and techniques to be applied. As this project is under development, it is expected that the results of this evaluation will allow the understanding of the repercussions of the project on the knowledge and attitudes of students of Higher Education, but also the impact of this project in classes of Primary Education, including children with and without disabilities, and their respective teachers.
\end{abstract}

Keywords: prolearn4all; qualitative evaluation; inclusion; Primary Education; ludic-pedagogical resources

\section{Evaluación Quantitativa del ProLearn4ALL: un Proyecto de Investigación Basado en la Resolución de Problemas}

Resumen: El ProLearn4ALL - Maletas Pedagógicas para TODOS, es un proyecto que tiene como principal objetivo la creación de recursos ludopedagógicos a los que puedan acceder niños del $1^{\circ}$ Ciclo de Enseñanza Básica (6-10 años), a fin de sensibilizarlos a la diferencia y, en particular, a los cuatro dominios de la discapacidad (intelectual, motora, auditiva y visual). Este proyecto tiene la particularidad de incluir en su equipo de trabajo diversos elementos, no solo docentes e investigadores, sino también estudiantes de pertenecientes a diferentes niveles de Enseñanza Superior (Cursos Técnicos Superiores Profesionales, Licenciaturas y Másteres) que se enfrentan a una secuencia de preguntas para las que tienen que encontrar respuestas y soluciones válidas. El aprendizaje basado en problemas representa, así, la estrategia seguida a lo largo de la investigación y formación, una estrategia que implica una evaluación sistemática del trabajo realizado. La evaluación de todo el proceso, de la fase de planificación a la de implementación, se describe en este artículo, reflejándose en las opciones tomadas en lo que se refiere a los instrumentos y técnicas que deben aplicar. Considerando que este proyecto está en curso, se espera que los resultados de esta avaluación permitirán comprender las repercusiones del proyecto sobre los conocimientos y aptitudes de los estudiantes de Enseñanza Superior, así como su impacto en las clases de Enseñanza Básica, que incluyen niños con y sin discapacidad, y en sus respectivos profesores.

Palavras clave: prolearn4all; evaluación cualitativa; inclusión; $1^{\circ}$ Ciclo de Enseñanza Básica; recursos ludopedagógicos.

\section{Évaluation Qualitative du ProLearn4ALL: un Projet de Recherche Basé sur la Résolution de Problèmes}

Résumé: ProLearn4ALL - Maletas Pedagógicas para TODOS est un projet dont le but principal est la création de ressources éducatives et ludiques accessibles à des enfants de l'école élémentaire, afin de les sensibiliser à la différence et en particulier aux quatre domaines de l'handicap (intellectuel, moteur, auditif et visuel). Le projet a la particularité d'inclure dans son équipe de travail plusieurs éléments, non seulement des professeurs et des chercheurs, mais également des étudiants de différents niveaux d'enseignement supérieur (enseignement supérieur technique professionnel, licence et maîtrise) confrontés à une série de questions pour lesquelles ils doivent trouver des réponses valables et des solutions. L'apprentissage par problème est ainsi devenu la stratégie suivie tout au long de la recherche et formation, ce qui implique une évaluation systématique du travail mise en place. L'évaluation de l'ensemble du processus, dès la planification à la phase de mise en œuvre, est décrite dans cet article, ayant lieu à une réflexion sur les options prises au niveau des instruments et des techniques à appliquer. Étant donné que ce projet est en cours, on prévoit que les résultats de cette évaluation permettront de comprendre les répercussions du projet sur les connaissances et les attitudes des étudiants de l'enseignement supérieur, mais également sur l'impact de ce projet sur les classes de l'enseignement de base, y compris les enfants handicapés et non handicapés, et leurs enseignants.

Mots-clés: prolearn4all; évaluation qualitative; inclusion; éducation élémentaire; ressources ludo-pédagogiques. 


\section{Introdução}

O ser humano pode ser confrontado, em qualquer momento, com uma deficiência ou limitação que afete a sua funcionalidade em diversos contextos da vida (World Health Organization, 2016). Na escola, muitos destes casos são denominados de $\mathrm{Ne}$ cessidades Específicas (NE). "In Europe, recent estimates place the number of children with special educational needs (SEN) at 15 million." (Drabble, 2013, p. 1) e, em Portugal, de acordo com a Direção-Geral de Estatísticas da Educação e Ciência, 87039 alunos apresentavam NE no ano letivo 2017/2018, dos quais 21426 frequentavam escolas do $1^{\circ}$ Ciclo do Ensino Básico.

O elevado número de alunos com NE justifica a necessidade das escolas assegurarem todas as condições necessárias à sua plena participação, nomeadamente pelo facto de se manterem, em pleno século XXI, situações de exclusão para com os que são diferentes. Nem todos são bem aceites num determinado grupo, alguns alunos com necessidades específicas continuam a ser socialmente excluídos, pertencendo a grupos vulneráveis (Krischler, Cate \& Krolak-Schwerdt, 2018).

Importa, neste sentido, que as escolas se tornem mais inclusivas, de forma a sensibilizar as pessoas para a diferença e concomitantemente para a sua aceitação. Esta necessidade tem dado origem à criação de uma série de legislação em muitos países, onde se inclui Portugal, denotando-se que as linhas orientadoras e as premissas da inclusão são consonantes, independentemente do país, quando advogam que "todos os alunos devem aprender juntos, sempre que possível, independentemente das dificuldades e diferenças que apresentem" (Declaração Salamanca, 1994, p.11), conscientes de todas as vantagens e dificuldades que tal situação possa implicar.

Para o efeito, é fundamental criar recursos e contextos acessíveis que não excluem pela diferença mas que a assumem como fator de enriquecimento do espaço escolar, enquanto reflexo da sociedade civil.

O projeto ProLearn4ALL segue, precisamente, estas premissas, ao procurar, através da resolução sequencial de problemas, criar produtos lúdico-pedagógicos aplicáveis em turmas regulares do $1^{\circ}$ Ciclo do Ensino Básico que incluam alunos com e sem NE.

Como qualquer projeto de investigação, o ProLearn4ALL inclui também a avaliação, dos processos e dos resultados, no sentido de ajustar práticas e analisar em que medida os resultados expectáveis foram atingidos. As opções metodológicas e as técnicas e instrumentos de avaliação foram conscientemente selecionados em função dos objetivos delineados para estudo, sendo descritos e devidamente fundamentados neste artigo. 


\section{Contextualização Teórica}

A escola inclusiva reconhece que a presença e aceitação de qualquer aluno, independentemente das suas características, enriquece a criança com NE, assim como todos os que com ela convivem, na medida em que estimula um conjunto de competências, conhecimentos e valores sociais (Drabble, 2013). De acordo com uma revisão de literatura sistemática realizada por Ulger, Dette-Agenmeyer, Reichle e Gaertner (2018) uma boa interação grupal nas escolas gera maior sentido de pertença dos alunos, meIhoria do seu bem-estar e, de uma forma geral, experiências educativas mais positivas, o que se traduz num melhor desempenho académico.

Apesar desta constatação, sabe-se que os alunos com necessidades específicas são menos aceites que os seus pares e pelos seus pares, continuando a observar-se uma grande diferença entre os ideais veiculados pela Declaração de Salamanca e a realidade praticada nas escolas (Ferreira, 2015). Os outros, os pares, são essenciais a qualquer processo de inclusão. A inclusão não será real se apenas se traduzir numa presença física da criança/jovem diferente em contexto escolar (Ülger, Dette-Hagenmeyera \& Reichlea, 2018). A ambição deve ser maior, o desafio será, também, o de incluir as crianças em todas as dimensões necessárias ao desenvolvimento do ser humano (Borges \& Coelho, 2015). A compreensão, aceitação e tolerância da diferença, independentemente da sua origem ou tipologia, devem ser comportamentos naturais, independentemente de ser adulto ou criança, devendo a tónica colocar-se nesta última, ajudando-a a perceber que o importante será sempre o ser humano como pessoa, com as respetivas caraterísticas.

A forma como os pares se relacionam e percecionam o outro, desde a infância, deve merecer a atenção de todos, considerando-se o impacte desta influência na vida dos indivíduos. Contudo, o facto de alguns estudos nos darem conta que "os pares dos alunos com NEES tendem a apresentar dificuldades em compreender/aceitar a diferença/deficiência, nomeadamente em contexto educativo [e] frequentemente os alunos de uma turma, ao lidarem com um/a colega que apresente NEES, tendem a revelar alguma intolerância face ao seu tratamento "diferenciado" (Borges \& Coelho, 2015, p. 16), dificulta o desenvolvimento holístico de todos. A possibilidade do aluno realizar as suas tarefas e atividades com a turma, utilizando os mesmos materiais pedagógicos poderá ser um fator de aproximação entre pares. Do lado do professor, o que faz a diferença é o modo como este seleciona e utiliza os materiais didático-pedagógicos para que permitam que todos os alunos atinjam os objetivos curriculares.

A escola inclusiva tem o potencial de alterar atitudes e valores perante a diferença, ao educar todas as crianças juntas (Ainscow, 2016). Esta escola inclusiva deve ter início numa fase precoce do desenvolvimento infantil, continuando até ao ensino secundário e mesmo superior. Na realidade, é possível encontrar, na literatura científica, 
evidências de que o aumento da idade da criança a torna menos flexível no que se refere à aceitação de outras crianças/jovens diferentes, seja dentro ou fora da escola. "Developmental and social psychological research has shown that children begin to reveal biases that favor their social group and prejudiced attitudes around the age of 5 years" (Ülger, Dette-Hagenmeyera \& Reichlea, 2018, p.88).

As escolas devem, portanto, adaptar-se, disponibilizando oportunidades às crianças para adquirir novas competências através de atividades e experiências significativas e relevantes. Estas devem incluir estratégias de sensibilização para a diferença, para que os alunos sejam informados sobre as especificidades dos domínios da deficiência (Drabble, 2013). Aumentando a consciência das crianças, e estando estas num momento propício à assimilação de novas informações, estamos a contribuir para educar melhores cidadãos que irão crescer fomentando hábitos e atitudes de inclusão.

Assume-se, portanto, que uma escola e sociedade inclusivas privilegiam as especificidades de cada indivíduo, sendo necessário criar condições para que a participação de todos seja efetiva, o que significa tornar os contextos acessíveis (Francisco et al, 2015). A acessibilidade será tanto mais efetiva quanto mais se tiver em conta o design universal na criação dos recursos, podendo estes ser utilizados pela generalidade das crianças e não apenas pelas que têm necessidades especiais.

Neste sentido, a criação de recursos e planeamento de atividades lúdicas é essencial para o desenvolvimento de múltiplas habilidades e funções da criança, no plano cognitivo, social, emocional e motor, servindo o propósito de ensinar-aprender uma multiplicidade de áreas úteis à vida quotidiana (Mendes, 2015). Desta forma, o professor titular deve ter em conta o modo como os seus alunos vão interagir com os recursos, para que se criem espaços relevantes de troca de experiências entre todos, nomeadamente com crianças com Necessidades Específicas.

O projeto ProLearn4ALL foi criado com este intuito, tendo como objetivo basilar aumentar o conhecimento das crianças do $1^{\circ}$ Ciclo do Ensino Básico (CEB) para a área das NE, de uma forma lúdico-pedagógica.

\section{O Projeto ProLearn4ALL: Maletas Pedagógicas para TODOS}

O projeto será desenvolvido através de uma parceria entre o Instituto Politécnico de Leiria (Escola Superior de Educação e Ciências Sociais; Escola Superior de Artes e Design), o Instituto Politécnico de Coimbra (Escola Superior de Educação), o Observatório da Inclusão e Acessibilidade em Ação - (CICS.NOVA.IPLeiria-iACT), o Laboratório de Investigação em Design e Artes; a Cooperativa de Ensino e Reabilitação de Crianças Inadaptadas e a Câmara Municipal de Leiria.

A criação de uma plataforma colaborativa, entre as diferentes instituições, tem permitido o desenvolvimento de dinâmicas de ensino, aprendizagem e reflexão através 
do trabalho interdisciplinar nas áreas da Educação + Design + Inclusão. Este processo, baseado na resolução de problemas, tem permitido a troca de conhecimentos e experiências entre diferentes escolas, cursos, unidades de investigação e respetivos estudantes, professores e investigadores, beneficiando as comunidades locais, no que se refere à inclusão social (Mangas, Freire \& Santos, 2018).

O ProLearn4ALL pretende desenvolver produtos lúdico-pedagógicos acessíveis que abordem os quatro domínios da deficiência (auditivo, intelectual, motor e visual), tendo em conta a escassez de recursos que potenciem a motivação e interesse para aprender de uma forma recreativa. Estes, desenvolvidos numa lógica de multiformato, adaptar-se-ão às diferentes características das crianças e às suas formas de ler o mundo, tendo como intenção o aumento de conhecimentos sobre as NE.

Neste âmbito, o projeto procura responder a algumas questões, nomeadamente:

- Que tipo de características devem ter os produtos pedagógicos de forma a se tornarem acessíveis a crianças do $1^{\circ} \mathrm{CEB}$ ?

- Quais os processos produtivos mais eficientes para criar produtos de alto valor, acessíveis e inovadores?

- De que forma a utilização dos produtos aumenta o conhecimento relativo às NE e promove a inclusão entre crianças do $1^{\circ} \mathrm{CEB}$ ?

De forma a responder a estas questões, foram delineados os seguintes objetivos: Recolher dados relativos a materiais lúdico-pedagógicos acessíveis existentes no mercado; Desenvolver propostas de produtos lúdico-pedagógicos inovadores e acessíveis relacionados com NE; Desenvolver o design e ilustração das propostas obtidas; Produzir protótipos de produtos lúdico-pedagógicos acessíveis, relativos às NE; Testar os protótipos de produtos lúdico-pedagógicos acessíveis (maleta) relativos às NE nas Crianças do $1^{\circ}$ ciclo e professores das escolas da $1^{\circ} \mathrm{CEB}$ dos agrupamentos de Escolas de Leiria; Reformular os produtos finais e validá-los com especialistas nas áreas da inclusão e acessibilidade e Disseminar o projeto; Aplicar a versão final da maleta aos alunos das escolas do $1^{\circ} \mathrm{CEB}$ dos agrupamentos de Escolas e Partilhar os resultados.

Sendo os recursos construídos aplicados e testados em turmas regulares do $1^{\circ}$ CEB, prevê-se o envolvimento de alunos com e sem NE, procurando contrariar a tendência da investigação levada a cabo na área da inclusão escolar. "Many of the existing interventions carried out in schools have tested effects that are related to majority children's attitudes toward ethnic minorities, but there is very little evaluated intervention work that has involved minority children as participants. Similarly, there is a preponderance of intervention research on attitudes toward persons with disabilities but no research involving these targets as participants." (Ülgera, Dette-Hagenmeyera \& Reichlea, 2018, p.100).

No projeto participam estudantes de diferentes níveis de Ensino Superior (ES) e áreas, tais como a educação, comunicação, ciências sociais, artes e design e língua 
gestual procurando-se avaliar o seu envolvimento no ProLearn4ALL. Espera-se, deste modo, promover o raciocínio crítico e competências de investigação científica nos nossos estudantes na medida em que o projeto assenta numa metodologia de aprendizagem (e de investigação) baseada em problemas. Esta considera-se a mais adequada, uma vez que permite o envolvimento direto de todos nas várias fases do projeto (design, produção, testes, reformulação e validação dos produtos lúdico-pedagógicos).

O Projeto ProLearn4ALL, em desenvolvimento, pretende, na etapa final, que os produtos reformulados e validados sejam reunidos num kit a oferecer às escolas do $1^{\circ}$ Ciclo do Ensino Básico dos Agrupamentos de Escolas de Leiria, para que possam ser utilizados pelos professores em qualquer momento do percurso escolar das crianças que acompanham. A sensibilização destas crianças levará, de forma indireta, à sensibilização dos próprios "pais, profissionais e todas as pessoas em geral para a necessidade de estruturar e adaptar atitudes, estratégias e materiais no sentido de minimizar as diferenças" (Gonçalves, 2017, p.61).

\section{Aprendizagem baseada em problemas, enquanto estratégia de aprendizagem ativa de estudantes de ensino superior}

A aprendizagem ativa assume que os contextos educativos não se cinjam à transmissão de conhecimentos, onde o estudante tem um papel passivo, mas sejam espaços onde o estudante tem um papel ativo na construção do seu próprio conhecimento (Mizokami, 2018). Desta forma, a aprendizagem torna-se num processo dinâmico baseado na aquisição de conhecimentos significativos, na medida em que o estudante está constantemente a criar e alterar relações entre os novos conhecimentos e os já existentes (Barkley, 2018).

Para que tal seja possível, é essencial reunir condições de trabalho que permitam aos estudantes interagirem uns com os outros e contextos de aprendizagem estimulantes através de tarefas desafiantes, criação de comunidades e orientação que possibilitem uma aprendizagem holística (Barkley, 2018). A aprendizagem ativa enfatiza, ainda, a importância da adequação das atividades a desenvolver com os processos cognitivos externos, de forma a beneficiar o mundo exterior (Mizokami, 2018). Ao se sentirem motivados, os estudantes comprometem-se mais com o seu processo de aquisição de conhecimento (Barkley, 2018).

A aprendizagem ativa proporciona uma reflexão sobre as mudanças societais, que decorrem no dia-a-dia, permitindo o desenvolvimento de competências variadas, tais como capacidades interpessoais e sociais (Mizokami, 2018), colocando o estudante num papel central, na medida em que constrói, reconstrói e cria a sua forma de compreensão dos conceitos (Barkley, 2018). 
Neste âmbito, no projeto ProLearn4ALL, a aprendizagem baseada em problemas surge como uma estratégia pedagógica com estudantes de ensino superior, que permite que estes aprendam através da resolução de uma determinada questão que se levante num contexto específico (Yew \& Goh, 2016). A procura de soluções para estes problemas requer o desenvolvimento de competências de pensamento crítico e criativo, o que permite a construção do conhecimento. Nesta estratégia o estudante tem um papel central, no que se refere à construção do conhecimento, sendo o professor um facilitador da aprendizagem, um orientador que direciona o trabalho (Ulger, 2018).

Yew e Goh (2016) consideram que a aprendizagem baseada em problemas permite a retenção do conhecimento e respetiva aplicação a longo prazo, na medida em que a pessoa é confrontada com uma determinada situação e tem de encontrar respostas adequadas. Esta abordagem permite a posterior criação de analogias em novos contextos onde deverá procurar a melhor forma de encontrar soluções que permitam resolver novos problemas.

Hallinger e Bridges (2016) apresentam um modelo de aprendizagem baseada em problemas, a partir de uma adaptação em contextos de gestão de educação, onde destacam seis grandes características:

1. O problema é o ponto de partida e não a teoria.

2. A aprendizagem baseia-se num problema que os estudantes podem vir a ter de enfrentar nas suas práticas profissionais futuras.

3. As unidades curriculares baseiam-se no problema e não nos conceitos disciplinares.

4. O estudante assume a responsabilidade na gestão da sua aprendizagem.

5. A aprendizagem decorre a partir de trabalhos em equipa orientados pelo professor e não dos processos de aulas expositivas.

6. Os estudantes apresentam potenciais soluções para o problema, recebendo feedback formativo do seu trabalho.

Ulger (2018) apresenta o processo cíclico da aprendizagem baseada em problemas relativo ao estudo experimental que realizou, mas que ilustra bem as várias fases do processo, pelo que se considera pertinente a partilha e respetiva análise.

Neste sentido, a Figura 1 reflete o carater cíclico do processo, o qual tem como ponto de partida a apresentação do problema. Segue-se a análise da realidade e das necessidades previstas, face ao problema proposto. Desta forma, é possível identificar qual a informação necessária, no sentido de efetuar pesquisas e discussão em grupo. Este carácter cíclico gera brainstormings de ideias que poderão culminar em sugestões para potenciais soluções. Durante todo este processo, o professor, enquanto orientador, coloca questões aos estudantes, ao invés de dar soluções, o que incentiva o pensamento criativo e reflexivo. 


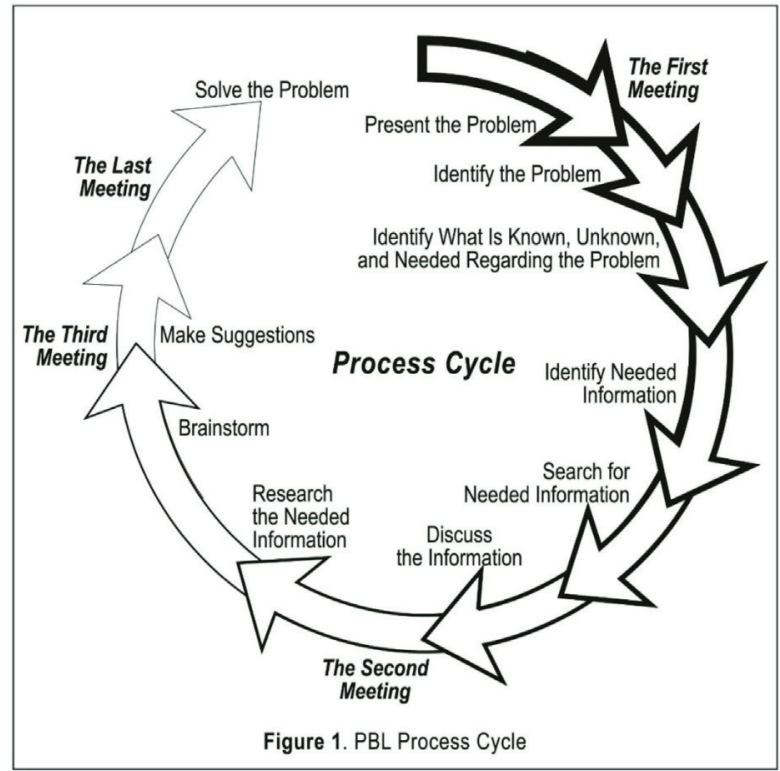

Figura 1. Processo cíclico da aprendizagem baseada em problemas (tradução própria de Ulger, 2018, s.p.)

\section{Metodologia}

Uma investigação científica deve incluir uma avaliação sistemática que permita analisar o cumprimento dos objetivos inicialmente previstos (Shek, 2017), garantindo o controlo de qualidade e possibilitando a sua melhoria e reorientação ao longo do processo.

A abordagem quantitativa faculta dados que permitem a identificação de tendências (Shek, 2017), contudo, as estratégias qualitativas possibilitam a exploração e compreensão de um dado fenómeno (Plano Clark \& Creswell, 2015) de uma forma holística, ao contrário dos métodos quantitativos (Mertens, 2015). No que se refere ao projeto ProLearn4ALL, a avaliação enquadra-se num paradigma essencialmente qualitativo, com o intuito de se compreender com maior profundidade o processo desenvolvido e os resultados alcançados, contribuindo para modificar e melhorar práticas (Mangas, Freire \& Santos, 2018).

A aprendizagem baseada em problemas, já explicada no corpo teórico, permite que o estudante seja confrontado com situações reais que necessitam de respostas, para as quais é exigido um pensamento crítico e autónomo (Yew \& Goh, 2016) já que envolve uma série de processos cognitivos. Tendo em conta estes pressupostos, 
optou-se por seguir esta abordagem, ao longo de todo o projeto, nomeadamente na sua avaliação, por se considerar ser a mais apropriada e a que melhor serve os objetivos que se pretendem alcançar.

\section{Avaliação do Projeto ProLearn4ALL}

O projeto ProLearn4ALL conta com uma equipa abrangente de professores, investigadores e especialistas de várias áreas do saber, e com a participação de estudantes de vários ciclos de estudos do ES, o que permite obter várias perspetivas de um mesmo problema, com vista à criação de diversas propostas de solução. Ao longo do processo os produtos lúdico-pedagógicos vão sendo ajustados em função das observações e intervenções dos vários estudantes que participam nas diferentes atividades, pelo que se trata de um processo iterativo e interativo, a partir do qual se procuram as respostas mais acessíveis à diversidade de públicos (Mangas, Freire \& Santos, 2018).

Com o intuito de se compreender em que medida o ProLearn4ALL poderá influenciar as atitudes e/ou comportamentos dos estudantes de ES e dos alunos de $1^{\circ} \mathrm{CEB}$, assim como o impacte do projeto, pretende-se fazer uma avaliação de todo o processo, dos resultados imediatos e a longo prazo relativos à respetiva implementação nas escolas de $1^{\circ} \mathrm{CEB}$.

\section{Avaliação do Processo}

O processo de criação de produtos lúdico-pedagógicos, incluindo a sua planificação, implementação e reformulação, conta com a participação de estudantes do ES, pelo que se torna necessário avaliar a sua envolvência no projeto, assim como o impacte do mesmo nas suas atitudes. Para o efeito, encontra-se em aplicação um inquérito por questionário com questões de resposta fechada e aberta. Este tipo de questões possibilita a obtenção de dados quantitativos que sustentem a literatura científica e, por outro lado, a exploração das opções das respostas fechadas (Plano Clark \& Creswell, 2015).

\section{Avaliação dos Resultados Imediatos}

Os produtos criados pelos estudantes do ES serão validados por consultores e testados em escolas do $1^{\circ} \mathrm{CEB}$.

Os especialistas externos, cuja perspetiva é mais distanciada, têm um olhar focado nos objetivos definidos para o projeto, nomeadamente na área da inclusão. Os seus pareceres serão registados numa grelha que permitirá validar as opções tomadas na 
construção dos produtos e identificar eventuais necessidades de reformulação de alguns elementos, no sentido de melhorar a sua acessibilidade.

Também no sentido de testar e melhorar a maleta, futuros professores do $1^{\circ} \mathrm{CEB}$, em contexto de prática pedagógica, irão aplicar os produtos, junto das crianças, procurando perceber a sua aceitação, envolvendo, de igual forma, os professores titulares das turmas. Para o efeito, os estagiários terão um papel de observadores e mediadores. A observação participante enriquece o estudo, pois permite que se obtenha uma perspetiva direta das intervenções de todos os envolvidos o que possibilita uma melhor compreensão e aprofundamento de uma dada situação (Plano-Clark \& Creswell, 2015).

O registo das observações será efetuado numa grelha semiestruturada, a qual foi escolhida pela riqueza dos dados que é possível obter, tendo em conta que permitem: analisar o ambiente e contexto envolvente; descrever as dinâmicas e comportamentos que possam ocorrer no momento; compreender os processos e relações entre as pessoas envolvidas numa determinada situação e identificar novos problemas, que por sua vez geram novas questões suscetíveis de investigação (Cohen, Manion \& Morrison, 2018).

A grelha inclui indicadores predefinidos que atestam o grau de aceitação dos novos produtos, a participação e o desempenho no decorrer das atividades, e contará com um espaço aberto para registo de elementos que possam não ter sido previstos.

Para além das grelhas de registo das observações, serão, também, aplicados inquéritos por questionário às crianças, no sentido de se conhecerem as dinâmicas resultantes da aplicação dos produtos lúdico-pedagógicos em sala de aula e de forma a possibilitar a triangulação de resultados. O instrumento a utilizar, deverá contemplar questões de respostas fechada, num formato gráfico mais apelativo, acessível e simples, considerando a idade e nível de compreensão leitora dos alunos.

A recolha da opinião dos professores titulares das turmas do $1^{\circ} \mathrm{CEB}$ será efetuada através de uma entrevista semiestruturada, com o intuito de se compreender em maior profundidade o contributo da aplicação dos produtos da maleta em contexto escolar. A entrevista, sendo um instrumento de recolha de dados flexível, possibilita a utilização de vários canais multissensoriais: verbal, não-verbal, falado e ouvido (Cohen, Manion \& Morrison, 2018), assim como o conhecimento das perceções das pessoas e a forma como um determinado evento pode afetar os seus pensamentos e sentimentos (Mann, 2016). A opção por uma entrevista semiestruturada deve-se à possibilidade de introdução de novas questões, não previstas inicialmente, que permitirão a obtenção de mais informação e consequente aprofundamento das temáticas tratadas (Plano-Clark \& Creswell, 2015), enriquecendo o estudo em questão. 


\section{Avaliação dos resultados a longo prazo}

De forma a analisar o efeito da aplicação das maletas numa perspetiva longitudinal, realizar-se-ão, após um ano, entrevistas em grupo aos professores titulares das turmas de $1^{\circ} \mathrm{CEB}$ onde será implementado o projeto. Esta técnica consiste numa conversa sistematizada com um pequeno grupo de pessoas que possibilita a interpretação da realidade a partir de processos de reflexão mútua com o outro (Caillaud \& Flick, 2017).

Cabe ao investigador, no focus group, também chamado de grupo de discussão (Silva, Veloso \& Keating, 2014), captar a perspetiva individual dos participantes sobre o tema em estudo, identificando as suas crenças, impressões e emoções, para além de opiniões e atitudes, o que permite obter o nível de concordância ou discordância dos mesmos face às perguntas levantadas (Lune \& Berg, 2017). Desta forma, colocando a mesma questão a diversos intervenientes, gera-se discussão, interação, cruzamento de ideias e levantamento de novas questões (Mertens, 2015), o que conduz à partilha e clarificação de pontos de vista. Esta dinâmica permite uma maior consciência crítica dos elementos e corresponsabilização face ao tema em discussão (Caillaaud \& Flick, 2017).

O procedimento implica várias fases, que vão desde o planeamento e preparação, passando pela moderação e, por fim, a análise de dados e divulgação de resultados (Silva, Veloso \& Keating, 2014), sendo fundamental a construção de um guião que servirá como indutor do processo de discussão/reflexão. Este deve inclui instruções para o processo de realização da entrevista, questões abertas que permitam a expansão do discurso dos participantes, que podem ou não ser todas colocadas em função da forma como a discussão evolui, e espaço para notas que advenham das respostas dos participantes sendo, portanto, um instrumento semiestruturado (Clark \& Creswell, 2015).

A aplicação deste instrumento, no projeto ProLearn4ALL, permitirá compreender a relevância atribuída pelos professores à utilização de produtos lúdico-pedagógicos acessíveis nas suas atividades letivas, o que pode contribuir para o aumento do seu interesse e envolvimento nas áreas da inclusão e acessibilidade (Mangas, Freire \& Santos, 2018).

\section{Análise de resultados}

Os dados provenientes dos diversos instrumentos utilizados permitirão a triangulação de resultados, o que por sua vez possibilitará a validação dos mesmos, assim como a obtenção de uma visão holística da avaliação de todo o projeto. Apesar de se tratar de uma análise essencialmente qualitativa, a utilização de dados quantitativos torna-se útil, na medida em que complementam esta avaliação com informação 
pertinente. Neste sentido, e para que seja possível conhecer a tendência das respostas, será efetuada uma análise descritiva simples, que será, posteriormente, aprofundada a partir dos dados advindos das questões de resposta aberta.

No que se refere aos dados qualitativos, estes serão alvo de análise de conteúdo, através de procedimentos sistemáticos de categorização e codificação mistos (Amado, Costa \& Crusoé, 2013). Desta forma, serão identificadas categorias e subcategorias decorrentes da revisão da literatura, podendo emergir algumas a posteriori, face ao corpus em análise.

Deste modo, os dados qualitativos permitirão a organização da informação textual, que dará lugar à identificação, de forma sistemática e objetiva, de indicadores/ unidades de registo e de contexto que, por sua vez, permitirão uma interpretação e inferência dos resultados mais pertinentes considerando os objetivos do projeto (Bardin, 2015).

\section{Considerações finais}

O artigo apresentado pretendeu delinear o processo de avaliação do projeto ProLearn4ALL que, numa lógica de resolução de problemas, pretende desenvolver e testar soluções acessíveis de recursos lúdico-pedagógicos destinados a TODAS as crianças do $1^{\circ}$ CEB. Neste âmbito, procurou-se definir e justificar, com base na literatura, os instrumentos e técnicas de recolha e análise de dados a adotar e os resultados expectáveis, a curto e longo prazo.

A avaliação permitirá compreender a influência das atividades desenvolvidas nos conhecimentos e práticas dos estudantes de ES. Espera-se que estes tenham um papel proativo na busca das soluções mais adequadas aos objetivos traçados inicialmente, o que contribui para o desenvolvimento das suas competências reflexivas e críticas, fundamentais nos contextos profissionais futuros.

Tendo o projeto a dupla vertente de criação e implementação dos produtos lúdico-pedagógicos, perspetiva-se, ainda, que os resultados se disseminem pela comunidade escolar do $1^{\circ} \mathrm{CEB}$. Para o efeito, importa obter resultados que possibilitem uma compreensão aprofundada do impacte que estes produtos possam ter nas crianças e professores e nas dinâmicas de inclusão em que se envolvem.

Tratando-se de um projeto ainda em curso, espera-se que este seja um passo relevante e estruturado para as etapas seguintes, possibilitando que os resultados alcançados possam gerar uma real mudança de atitudes das crianças e jovens a quem o projeto se destina, e dos próprios profissionais de educação que os acompanham. 


\section{Agradecimentos}

Projeto cofinanciado pelo FEDER - Fundo Europeu de Desenvolvimento Regional, no âmbito do Programa Portugal 2020, através do CENTRO2020 - Programa Operacional Regional do Centro.

\section{Referências}

Amado, J., Costa, A.P. \& Crusoé, N. (2013). IV - 1. A Técnica da análise de conteúdo. In J. Amado (Coord.) Manual de investigação qualitativa em educação (pp. 299-349). Coimbra: Imprensa da Universidade de Coimbra.

Ainscow, M. (2016). Diversity and Equitity: A Global Education Challenge. New Zealand Journal of Educational Studies, 51(2), 143-155.

Bardin, L. (2015). Análise de conteúdo. Lisboa: Edições 70.

Barkley, E. F. (2018). Terms of engagement: understanding and promoting student engagement in today's College classroom. In K. Matsushita (ed.) Deep Active Learning: Toward greater depth in University Education. (pp. 35-57). Singapura: Springer Nature.

Borges, I. C. N. \& Coelho, A. M. S. (2015). O papel dos pares na inclusão de alunos com NEE: programa PARES. Exedra. Revista Científica. - http://www.exedrajournal.com/wp-content/ uploads/2016/02/01.pdf acesso 03/11/2018.

Caillaud, S \& Flick, U. (2017). Focus Group in triangulation contexts. In R. S. Barbour \& D. L. Morgan (Ed) A new era in Focus Group: Challenges, Innovation and Practice (pp. 155-177). United Kingdom:Palgrave macmillan.

Clark, V. \& Creswell, J. (2015). Understanding Research - A Consumer's Guide. New Jersey: Pearson Education.

Cohen, L., Manion, L. \& Morrison, K. (2018). Research methods in education (8 ${ }^{\mathrm{a}}$ Ed.). Oxon: Routledge Taylor \& Francis Group.

Direção-Geral de Estatísticas da Educação e Ciência - Necessidades Especiais de Educação 2016/2017 - http://www.dgeec.mec.pt/np4/224/ acesso 04/02/2018.

Drabble, S. (2013). Support for Children with Special Educational Needs (SEN). European Union: RAND Europe.

Ferreira, W. (2015). 20 Anos Depois de Salamanca...Onde Estamos e Para Onde Vamos? Poíesis Pedagógica, 13, (1), 87-106.

Francisco, M., Sousa, N., Esperança, C., Rodrigues, V. \& Neves, J. (2015). Práticas para um elearning acessível no Instituto Politécnico de Leiria. In C. Mangas, C. Freire \& M. Francisco (Orgs.), Inclusão e Acessibilidade em Ação Diferentes percursos, um rumo. Leiria: iACT/IPLeiria.

Gonçalves, H. (2017). Linguagem e perceção visual como meio de comunicação em crianças com perturbações do espectro de autismo. Dissertação de Mestrado em Design Gráfico. ESAD, IPLeiria.

Hallinger, P. \& Bridges, E. M. (2016). A systematic review of research on the use of problem-based learning in the preparation and development of school leaders. Educational Administration Quarterly, 1, 1-34. 
Krischler, M., Cate, I. M. P. \& Krolak-Schwerdt, S. (2018). Mixed stereotype content and attitudes towards students with special educational needs and their inclusion in regular schools in Luxembourg. Research in Development Disabilities, 75, 59-67.

Lune, H. \& Berg, B. (2017). Qualitative Research Methods for the Social Sciences. Long Beach: Pearson.

Mangas, C.; Freire, C. \& Santos, O. (2018). ProLearn4ALL Maletas Pedagógicas para TODOS: Estratégias e metodologias de avaliação qualitativa. In P. Costa; D. Souza; P. Castro; R. Saavedra \& S. Sá, Atas do $7^{\circ}$ Congresso Ibero-Americano em Investigação Qualitativa: Investigação Qualitativa na Educação (pp. 720-728). Oliveira de Azeméis: Ludomedia.

Mann, S. (2016). The research interview: reflective practice and reflexivity in research processes. UK: Palgrave Macmillan.

Mendes, M. (2015). A importância da ludicidade no desenvolvimento de crianças autistas. Instituto de Psicologia. Brasília: Universidade de Brasília.

Mertens, D. M. (2015). Research and evaluation in education and psychology: integrating diversity with quantitative, qualitative, and Mixed Methods (4th edition). USA: SAGE Publications, Inc.

Mizokami, S. (2018). Deep active learning from perspectives of active learning theory. In K. Matsushita (ed.) Deep Active Learning: Toward greater depth in University Education, 79-91. Singapura: Springer Nature.

Plano-Clark, V.L. \& Creswell, J. W. (2015). Understanding Research: a Consumer's guide (2nd edition). USA: Pearson Education, Inc.

Shek, D. T. L. (2017). The use of Focus Group in Programme Evaluation: Experience based on the project P.A.T.H.S. in Chinese Context. In R. S. Barbour \& D. L. Morgan (Ed) A new era in Focus Group: Challenges, Innovation and Practice (pp. 129-153). United Kingdom: Palgrave macmillan.

Silva, Veloso \& Keating (2014). Focus group: Considerações teóricas e metodológicas. Revista Lusófona de Educação, 26, 175-190. Disponível em http://revistas.ulusofona.pt/index.php/rleducacao/article/ view/4703, consultado em 3-01-2019.

Ulger, K. (2018). The effect of problem-based learning on the creative thinking and critical thinking disposition of students in visual arts education. Interdisciplinary Journal of Problem-Based Learning, 12(1) - https://doi.org/10.7771/1541-5015.1649 acesso 18/10/2018.

Ülger, Z., Dette-Agenmeyer, D. E., Reichle, B. \& Gaertner, S. L. (2018). Improving outgroup attitudes in schools: a meta-analytic review. Journal of School Psychology, 67, 88-103.

UNESCO (1994). Declaração de Salamanca sobre princípios, políticas e práticas na área das necessidades educativas especiais. UNESCO: Paris.

World Health Organization (2016). Internacional Classification of Functioning, Disability and Health https://www.who.int/classifications/icf/en/ acesso 16/01/2019.

Yew, E. H. J. \& Goh, K. (2016). Problem based learning: An overview of its process and impact on learning. Health Professions Education, 2, 75-79. 
Catarina Mangas

CICS.NOVA.IPLeiria - iACT; CI\&DEI

Escola Superior de Educação e Ciências Sociais - Politécnico de Leiria

Email: catarina.mangas@ipleiria.pt

ORCID: https://orcid.org/0000-0003-0843-5861

Carla Freire

CI\&DEl; CICS.NOVA.IPLeiria - iACT

Escola Superior de Educação e Ciências Sociais - Politécnico de Leiria

Email: carla.freire@ipleiria.pt

ORCID: https://orcid.org/0000-0001-7557-9596

Olga Santos

Cl\&DEl; CICS.NOVA.IPLeiria - iACT

Escola Superior de Educação e Ciências Sociais - Politécnico de Leiria

Email: olga.santos@ipleiria.pt ORCID: https://orcid.org/0000-0001-9119-9278

Correspondência

Catarina Mangas

Escola Superior Educação e Ciências Sociais

Campus 1

Rua Dr. João Soares

Apartado 4045

2411-901 Leiria - Portugal

Data de submissão: Feveveiro de 2018

Data de avaliação: Outubro de 2018

Data de publicação: Julho de 2019 